\title{
Adhesion of metal brackets to glassy matrix and hybrid CAD/CAM materials after
} different physico-chemical surface conditioning

\author{
Dilber, Erhan ; Aglarcı, Cahide ; Akın, Mehmet ; Özcan, Mutlu
}

DOI: https://doi.org/10.1080/01694243.2016.1159035

Posted at the Zurich Open Repository and Archive, University of Zurich ZORA URL: https://doi.org/10.5167/uzh-128058

Journal Article

Accepted Version

Originally published at:

Dilber, Erhan; Aglarcı, Cahide; Akın, Mehmet; Özcan, Mutlu (2016). Adhesion of metal brackets to glassy matrix and hybrid CAD/CAM materials after different physico-chemical surface conditioning. Journal of Adhesion Science and Technology, 30(15):1700-1709.

DOI: https://doi.org/10.1080/01694243.2016.1159035 
Adhesion of metal brackets to glassy matrix and hybrid CAD/CAM materials after different physico-chemical surface conditioning

\title{
Erhan Dilber, DDS, PhDa / Cahide Aglarcı, DDS, $\mathrm{PhD}^{\mathrm{b}} /$ Mehmet Akın, DDS, $\mathrm{PhD}^{\mathrm{c}} /$ Mutlu Özcan, DDS, Dr.med.dent., PhD $^{d}$
}

\begin{abstract}
${ }^{a}$ Assistant Professor, Sifa University, Faculty of Dentistry, Department of Prosthodontics, Izmir, Turkey ${ }^{b}$ Assistant Professor, Sifa University, Faculty of Dentistry, Department of Orthodontics, Izmir, Turkey ${ }^{c}$ Assistant Professor, Selcuk University, Faculty of Dentistry, Department of Orthodontics, Konya, Turkey ${ }^{d}$ Professor, University of Zurich, Dental Materials Unit, Center for Dental and Oral Medicine, Clinic for Fixed and Removable Prosthodontics and Dental Materials Science, Zurich, Switzerland
\end{abstract}

Short title: Adhesion of brackets to CAD/CAM materials.

Correspondance to: Prof. Dr. med. dent. Mutlu Özcan, University of Zürich, Dental Materials Unit, Center for Dental and Oral Medicine Clinic for Fixed and Removable Prosthodontics and Dental Materials Science, Plattenstrasse 11, CH-8032, Zürich, Switzerland. Tel: +41-44-63 45600, Fax: +41-44-63 44305. e-mail: mutluozcan@hotmail.com 


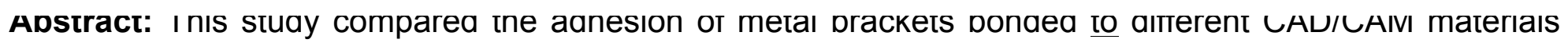
after various surface conditioning methods. CAD/CAM blocks ( $\mathrm{N}=204, \mathrm{n}=17$ per group) of a) VITA Mark II (VM), b) IPS e.max CAD (IP), c) Lava Ultimate (LU), and d) VITA ENAMIC (VE) were conditioned with one of the following methods: C-Control: (fine diamond bur); CJ: (fine diamond bur+air-abrasion with $30 \mu \mathrm{m}$ $\mathrm{SiO}_{2}+$ silane), and $\mathrm{HF}$ : (fine diamond bur+9.5\% hydrofluoric acid+silane). Metal brackets were bonded to the conditioned surfaces of the specimens, stored in artificial saliva for $24 \mathrm{~h}$ at $37^{\circ} \mathrm{C}$ and thermocycled (x1000). Subsequently, the brackets were debonded under shear in a Universal Testing Machine (1 $\mathrm{mm} / \mathrm{min}$ ). Failure types were analyzed under scanning electron microscope. Data were analyzed using two-way ANOVA and Tukey`s tests $(\alpha=0.05)$. Two-parameter Weibull distribution values, including the Weibull modulus, scale, and shape, were calculated. Mean bond strength (MPa) values were significantly affected by the surface conditioning method $(p<0.001)$ but not the CAD/CAM material type $(p=0.052)$. Bond strengths for all $\mathrm{CJ}$ and HF-conditioned specimens were two-fold higher $(11.83 \pm 1.95-9.44 \pm 1.63)$ than those for control specimens with all materials $(4.73 \pm 0.93-6.02 \pm 0.69)$. Lower mean values were obtained in LU-CJ $(9.78 \pm 1.61)$ and LU-HF $(9.44 \pm 1.63)$ than those for other groups (11.83 $\pm 1.95-$ $10.93 \pm 1.33)$ groups $(p<0.05)$. Weibull distribution showed higher shape values for VM-CJ (11.26) and VMHF (8.87) than those for other groups (0.82-1.83). In control groups, exclusively adhesive and after conditioning mainly mixed failures were observed. Chairside silica coating or HF conditioning significantly improved metal bracket adhesion to both glassy matrix and hybrid CAD/CAM materials tested, with the most reliable adhesion being observed for feldspathic ceramic.

Keywords: Adhesion; bond strength; CAD/CAM restorative materials; hydrofluoric acid; Lava Ultimate; lithium disilicate; silica coating; surface conditioning; VITA Enamic 
Industrially fabricated ceramic blocks are being increasingly used for restorations in adult patients with aesthetic and functional demands. Meanwhile, bonding of orthodontic brackets to CAD/CAM restorations has become more challenging with the steady increase in the number of adult patients seeking orthodontic treatment [1]. Intraoral polishing of a restoration is crucial in orthodontics since often glaze is removed after bracket debonding [2]. Typically, one-step CAD/CAM restorations can be manually polished, while twostep restorations made of lithium disilicate glass-ceramics can be manually polished or glazed [3].

Mechanical, chemical conditioning methods or a combination of them both have been suggested for ceramic surfaces in order to enhance adhesion of resin cements [4,5]. Hydrofluoric acid (HF) etching is a chemical conditioning method that creates surface roughness through preferential dissolution of the glassy phase from the ceramic matrix [6]. Subsequent surface conditioning of the etched ceramic surface with silane coupling agent enhances wettability and results in the formation of covalent bond with both the ceramic and resin cement [7]. However, HF is a highly toxic chemical, and etching silica-based ceramics produces insoluble silica-fluoride salts as by-products on the ceramic surface $[8,9,10]$. Despite this disadvantage, HF acid etching is routinely used for orthodontic bonding prior to bonding brackets to various ceramics $[4,11,12]$. The bond strength of resin composite to feldspathic ceramic is higher with $\mathrm{HF}$ etching followed by silanization than without $[13,14]$. An alternative to HF is tribochemical silica coating that is based on labside (Rocatec system, $110 \mu \mathrm{m}, 3 \mathrm{M}$ ESPE, St. Paul, MN, USA; ) or charside (CoJet, 30 $\mu \mathrm{m}, 3 \mathrm{M}$ ESPE) particle deposition methods using silica-coated alumina particles followed by silane application $[1,15,16,17]$. In the process of tribochemical silica coating, the substrate surface absorbs the kinetic energy of the particle and melts on the surface at $1200^{\circ} \mathrm{C}$ [18]. Embedded silica coated alumina particles leave the surface partially coated with silica and facilitate covalent bonding between the silica coated substrate and the resin composite. However, silica coating is not widely used for bonding orthodontic brackets to ceramic surfaces [4].

CAD/CAM blocks of conventional feldspathic silicate ceramic [19], resin-based nanocomposite [20], lithium disilicate glass-ceramic, as well as polymer-infiltrated ceramic network (PICN) (hybrid ceramic) [21, 22] are typically differed from each other according to their microstructure. While feldspathic silicate and lithium disilicate glass ceramics contain mostly mixture of feldspathic crystalline or lithium disilicate particles embedded in a glassy matrix, hybrid ceramics contain porous feldspathic ceramic matrix, 
infiltrated with a copolymer (urethane dimethacrylate and triethylene glycol dimethacrylate) [21]. Resinbased nano composites contain nanoceramic particles bound in the resin matrix. Hence they have quite different microstructure compared to ceramics [20].

To the best of our knowledge, adhesion of metal brackets to CAD/CAM materials in conjunction with surface conditioning methods has not been studied to date.

The objectives of this study therefore were to evaluate the adhesive potential of metal brackets to glassy matrix or hybrid CAD/CAM materials after various surface conditioning methods and to evaluate the failure modes after debonding. The null hypotheses tested were that CAD/CAM and conditioning method would not affect the adhesion of metal brackets.

\section{Materials and Methods}

Specimen preparation

Considering four different CAD/CAM materials and three surface conditioning methods for each group, power analysis using $\mathrm{G}^{*}$ Power [23] indicated an actual power value of $80 \%$, with 17 specimens per group, on the basis of the following: effect size $(f=1), \alpha=.005$; power: 80 ; non-centrality parameter: 15 ; and critical t: 5 .

CAD/CAM discs ( $N=204, n=51$ per group) of feldspathic ceramic VITA Mark II (VM), lithium disilicate glass-ceramic IPS e.max CAD (IP), resin-based nanocomposite Lava Ultimate (LU), and interpenetrating network ceramic VITA ENAMIC (VE) (Table 1), were fabricated (diameter: $6 \mathrm{~mm}$; thickness: $3 \mathrm{~mm}$ ) from their ingots using CEREC inLab MC X5 (Sirona Dental Systems GmbH, Bensheim, Germany). The milled specimen surfaces were finished with 1200 grit silicon carbide abrasive papers (3M ESPE) under water irrigation, followed by ultrasonic cleaning (Biosonic JR, Coltene, Whaledent) in distilled water for 3 min. The specimens of LU and VE materials were polished with their own polishing systems, while specimens of IP and VM were glazed according to each manufacturer's recommendations. The specimens were partially embedded in autopolymerizing acrylic resin blocks (Leaddent SC, Atlas Enta Dişçilik Ltd., İzmir, Turkey), with the glazed or polished surface exposed, and randomized into three groups according to one of the surface conditioning methods:

Surface conditioning protocols 


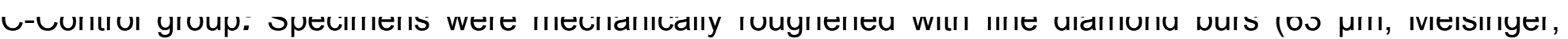
Hager\&Meisinger $\mathrm{GmbH}$, Neuss, Germany) placed with their shafts parallel to the specimen axes. Then, they were washed and rinsed thoroughly to remove the debris and air-dried.

CJ group: Following mechanical roughening $(63 \mu \mathrm{m})$, specimens were air-abraded with $30 \mu \mathrm{m}$ alumina particles coated with silica (CoJet Sand, 3M ESPE) using an intraoral air-abrasion device from a distance of approximately $10 \mathrm{~mm}$ at a pressure of 2.5 bar for $4 \mathrm{~s}$ [15]. The particle remnants were gently air-blown. Then, one coat of silane primer (Monobond Plus, Ivoclar Vivadent) was applied on the conditioned substrates, and waited for its reaction for $60 \mathrm{~s}$.

HF group: Following mechanical roughening $(63 \mu \mathrm{m})$, specimens were etched with $9.5 \% \mathrm{HF}$ gel (Ultradent Products Inc., South Jordan, UT, USA) for $90 \mathrm{~s}$ and washed with water for $60 \mathrm{~s}$. The conditioned substrates were then silanized as described in CJ group.

Bonding protocols

All bonding procedures were performed one clinician (C.A.) in accordance with the manufacturer's instructions. Metallic brackets (Kirium 3M Abzil, Sumaré, SP, Brazil) for maxillary premolars were bonded with resin cement (Transbond XT, 3M Unitek, Monrovia, California, USA) assigned for orthodontic purposes on the conditioned surfaces and photo-polymerized for $40 \mathrm{~s}$ from each direction using an LED (Great, Great Dental, Istanbul, Turkey) polymerization unit with a light intensity of $1000 \mathrm{~mW} / \mathrm{cm}^{2}$. The bracket was placed onto the substrate surface by using a bracket plier under static load of $100 \mathrm{~g}$. Excess cement was removed using microbrushes. The specimens were then stored in artificial saliva incubated at $37^{\circ} \mathrm{C}$ for $24 \mathrm{~h}$, and thermocycled $\left(1000\right.$ cycles, $\left.5-55^{\circ} \mathrm{C}\right)$ (Thermal Cycler Tester, DentalTeknik, Konya, Turkey).

Bond test and failure type analysis

The bonded interfaces were subjected to shear loading in a Universal Testing Machine (TSTM 02500, Elista Elektronik Informatik Sistem Tasarim Ltd., Istanbul, Turkey) at a crosshead speed of $1 \mathrm{~mm} / \mathrm{min}$. The force required to dislodge the bracket was recorded in Newton $(\mathrm{N})$ and then divided by the bracket surface area $\left(11.21 \mathrm{~mm}^{2}\right)$ to obtain bond strength values in megapascals (MPa).

After debonding, failure modes were analyzed using an optical stereomicroscope (Olympus SZ 40, Olympus Optical Co. Ltd, Tokyo, Japan) at x20 and categorized according to modified Adhesive Remnant Index (ARI) as follows: Score 0: All adhesive resin remained on the substrate surface; Score 1: $90 \%$ of 


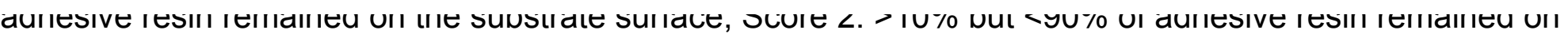
the substrate surface; Score 3: $<10 \%$ of the adhesive remained on the substrate surface, Score 4: No adhesive resin remained on the substrate surface; Score 5: Cohesive failure in the substrate material. One specimen from each group, representing each failure mode was sputter-coated with gold-alladium alloy (Cressington sputter coater 108auto, Cressington MTM-20, Elektronen-Optik-Service, Dortmund, Germany) and observed under Scanning Electron Microscopy (SEM; Evo LS10, Carl Zeiss, Oberkochen, Germany) at x20.

Statistical analysis

All statistical procedures were performed using the Statistical Package for Medical Science (SPSS Ver.11 for Windows, Chicago, IL, USA). Descriptive statistics were computed, and test of normality was performed using Kolmogorov-Smirmov and Shapiro-Wilk's tests. The means of each group were analyzed by two-way analysis of variance (ANOVA) and Tukey's post hoc test to compare the significant differences between the groups where the bond strength was the dependent variable and CAD/CAM materials (4 levels) and surface conditioning methods (3 levels) independent variables. Maximum likelihood estimation without a correction factor was used for two-parameter Weibull distribution, including the Weibull modulus, scale (m), and shape $\left({ }^{\circ}\right.$ ), to interpret predictability and reliability of adhesion (Minitab Software V.16, State College, PA, USA). $P$ values less than 0.05 were considered to be statistically significant in all tests.

\section{Results}

Mean bond strength (MPa) values were significantly affected by the surface conditioning method $(p<0.001)$ but not the CAD/CAM material type $(p=0.052)$ (Table 2$)$.

Bond strengths for all $\mathrm{CJ}$ and HF-conditioned specimens were two-fold higher $(11.83 \pm 1.95-9.44 \pm 1.63)$ than those for control specimens with all materials $(4.73 \pm 0.93-6.02 \pm 0.69)$ (Table 3$)$. Significantly lower mean values were obtained in LU-CJ $(9.78 \pm 1.61)$ and LU-HF $(9.44 \pm 1.63)$ than those for other groups $(11.83 \pm 1.95-10.93 \pm 1.33)$ groups $(p<0.05)$.

Weibull distribution showed higher shape values for VM-CJ (11.26) and VM-HF (8.87) than those for other groups (0.82-1.83) (Table 3).

The control specimens exhibited primarily Scores of 2 and 4, while all conditioned specimens showed Scores of 0 and 2. Cohesive failures were only observed in VE-HF and VE-CJ groups (Table 4). 


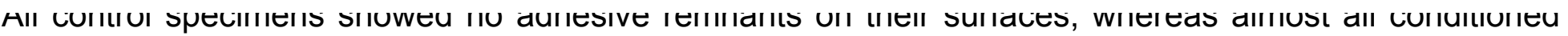
specimens showed adhesive remnants on most part of their surfaces (Figs. 2a-f).

\section{Discussion}

This study evaluated the adhesion of metal brackets on glassy matrix or hybrid CAD/CAM materials after various surface conditioning methods with a specific emphasis on HF etching and silica coating and silanization. Based on the results obtained, not the CAD/CAM material but the surface conditioning method significantly affected the results. Thus, the null hypotheses could be partially rejected.

In orthodontics, after the whole course of the treatment, the brackets need to be debonded from the restoration surfaces, preferably without damaging the substrate materials. For this reason, extremely good bond results are not desirable. Typically, bond strength values of 6-8 MPa are clinically adequate for metal brackets bonded onto enamel surfaces [24]. On the other hand, when the bond strength between the ceramic and resin composite exceeds $13 \mathrm{MPa}$, cohesive failures may occur within the ceramic [25] which then needs to be repaired intra- or extraorally. Therefore, semipermanent bond strength is expected being sufficiently high to resist accidental debonding during treatment, and sufficiently low to prevent the application of excessive forces during debonding the brackets [26]. In the present study, the mean bond strength values of metal brackets bonded to CAD/CAM surfaces generally exceeded acceptable limits except for control specimens. Also, except only a few cohesive failures in VE-HF and VE-CJ groups, no cohesive failures were experienced in the rest of the experimental groups.

In this study, the glaze or the polished top layers of materials were finished with a fine diamond bur. The reason for this is that HF would not be effective on the glaze and silane would not react with the surface to enhance bonding [24]. In this study, the polished surfaces of VE and LU, and the glazed surfaces of VM and IP, representing the control group, were removed by a fine diamond bur (63 $\mu \mathrm{m})$. Roughening with diamond burs without silane application was shown to decrease the bond strength compared to roughening with $\mathrm{HF}$ acid etching and silane application or $\mathrm{Al}_{2} \mathrm{O}_{3}$ air-abrasion and silane application alone [27]. Thus, considering also the low control group results, initial mechanical roughening is crucial prior to bracket bonding. Nevertheless, the obtained results in the control group, did not exceed values for the glassy-matrix ceramics (e.max: $11 \mathrm{MPa}$; Vita Mark: $5.4 \mathrm{MPa}$ ) according to the literature [24]. Previous studies found that metal brackets bonded to feldspathic porcelain with a silica coating or HF etching 


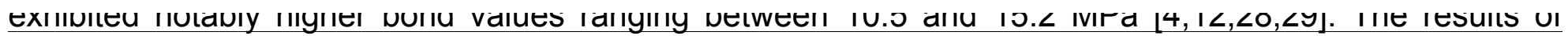
roughening with HF acid etching or silica coating and silane application of VM are consistent with the published literature (9.81 and 10.82 MPa, respectively) $[4,12,28,29]$.

It has to be noted that in this study a new silane was used. Silane primers commonly comprise 3Methacryloxyproyltrimethoxysilane (MPS) and are usually applied in the prehydrolyzed state in a solvent mixture of water and ethanol. These primers are commercially available in the dental market as single- or two-phase systems. While single-phase prehydrolyzed solutions exhibit a shorter shelf life compared to their two-phase counterparts, their ease of application plays an important role in the clinic setting [13]. Two-phase systems comprise hydrolyzed silane in ethanol and a hydrated acetic acid solution that are mixed to facilitate hydrolysis of the silane at a low pH before use [30]. Recently introduced universal silane primers (i.e. Monobond Plus, Ivoclar Vivadent, Schaan, Liechtenstein) combines three different functional methacrylates - silane methacrylate, phosphoric methacrylate, and sulfide methacrylate - in a single bottle [31].

Earlier studies identified HF etching or silica coating and silanization, as the most favorable conditioning methods regardless of the ceramic type namely, feldspathic, leucite-reinforced, lithium disilicate, fluoroapatite [4, 11]. However, the choice of conditioning method in the field of orthodontics remains controversial. Due to potential harmful effects of HF used in vivo, orthodontists prefer a safer alternative technique in order to improve adhesion of brackets to ceramic surfaces. In this study, VE, a hybrid ceramic containing feldspathic porcelain (86 weight\%) and an interpenetrating polymer network (14 w\%) [32] and $\mathrm{LU}$, a resin-based nanocomposite containing a combination of aggregated $20 \mathrm{~nm}$ silica and 4-11 $\mathrm{nm}$ zirconia clusters in a resin matrix were used [33]. The hardness of these materials are much lower than those for VM (10.64 $\pm 0.46 \mathrm{GPa})$ and VM9 $(9.5 \pm 0.35 \mathrm{GPa})$ [34]. Excessive wear of the opposing teeth can be prevented by the use of hybrid ceramics with lower hardness values instead of conventional feldspathic ceramics, particularly in adolescent patients [22]. Furthermore, hybrid ceramics are claimed to exhibit indentation creep responses similar to those of human enamel and superior stress redistribution [35]. Polymer pressure during processing and the density of preinfiltrated ceramic influence the final properties of the materials [22]. In the present study, the only few cohesive failures were observed in VE-CJ and VECJ groups. It is possible that both the organic and inorganic components of this material profited from both surface conditioning. 


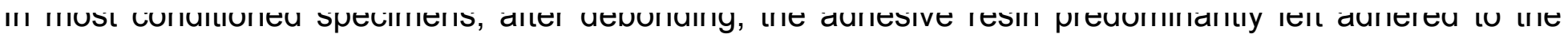
substrate surface (ARI Scores 0-2) indicating that the physico-chemical adhesion between the ceramic surface and resin cement exceeded the strength of the mechanical interlocking provided by the mesh bracket base. The lack of cohesive failures in general indicates that the bond strength achieved, did not exceed those of the CAD/CAM materials. Furthermore, this also shows that all materials presented strength higher than the cohesive strength of the luting adhesive resin. On the other hand, the control specimens mostly exhibited ARI Scores of 4 , with occasional scores of 2 or 3 , with no adhesive resin remaining on the surfaces after debonding. This implied a weak physic-chemical adhesion between the ceramic and the luting cement. Clinically, failures at the ceramic-composite interface are preferred through which, ceramic fractures and also extensive polishing procedures are avoided after debonding [36].

Bond strength of a material with a $5 \%$ chance of failure has been suggested to be at least 5.4 MPa [37]. In this study, the results for a $5 \%$ probability of failure in all conditioned specimens were higher than the proposed 5.4 MPa, while those for the control specimens did not present acceptable values to be safely used in the oral environment. The reliability of adhesion between the CAD/CAM materials and the brackets in conjunction with the studied conditioning methods were further investigated with the Weibull analysis, which provides information regarding the probability of bracket failure [38]. The results indicated drastically higher (almost 8 to 10-fold) reliability with the feldspathic ceramic with both condition methods that signifies less reliable results with all new CAD/CAM materials (0.82-1.83) tested.

This study provided information on adhesion to new CAD/CAM materials and independent of their chemical compositions, silica coating and HF etching enhanced the bonding of metal brackets followed by silane application. Simulating the complex nature of the oral environment is not possible due to variations in temperature, stresses, humidity, acidity, and plaque but the results need to be verified in in situ or clinical studies.

\section{Conclusions}

From this study, the following could be concluded:

1. Compared to grinding the surface with fine diamond bur only (control), all CAD/CAM materials tested benefitted from additional surface conditioning either with hydrofluoric acid or silica coating and silanization. 
2. Weibull parameters indicated more reliable adhesion of metal brackets to feldspathic ceramic when their graze was removed with fine diamond bur and then conditioned with either hydrofluoric acid or silica coating followed by silanization compared to those of other material-conditioning combinations.

3. The adhesive resin mostly remained on the surfaces of CAD/CAM materials subjected to hydrofluoric acid or silica coating, with no cohesive failures in most specimens.

\section{Clinical Relevance}

When metal brackets are to be bonded to CAD/CAM made reconstructions, orthodontists could achieve the most reliable adhesion to feldspathic ceramic after removing the glaze with fine diamond but and subsequently etching either with hydrofluoric acid or silica coating and silanization. If the orthodontist is unaware of the CAD/CAM restoration material, prior to bonding the metal bracket, application of one of these conditioning methods is essential.

\section{Conflict of interest}

The authors did not have any commercial interest in any of the materials used in this study.

\section{Acknowledgements}

This study was supported by Sifa University Coordination of Scientific Research Projects under Grant no. [2014-07]. 
[1] Sarac YS, Kulunk T, Elekdag-Turk S, Sarac D, Turk T. Effects of surface-conditioning methods on shear bond strength of brackets bonded to different all-ceramic materials. Eur J Orthod 2011;33:667-672. [2] Karan S, Toroglu MS. Porcelain refinishing with two different polishing systems after orthodontic debonding. Angle Orthod 2008;78:947-953.

[3] Mormann WH, Stawarczyk B, Ender A, Sener B, Attin T, Mehl A. Wear characteristics of current aesthetic dental restorative CAD/CAM materials: two-body wear, gloss retention, roughness and Martens hardness. J Mech Behav Biomed Mater 2013;20:113-125.

[4] Karan S, Buyukyilmaz T, Toroglu MS. Orthodontic bonding to several ceramic surfaces: are there acceptable alternatives to conventional methods? Am J Orthod Dentofacial Orthop 2007;132:144 e147114.

[5] Abu Alhaija ES, Al-Wahadni AM. Shear bond strength of orthodontic brackets bonded to different ceramic surfaces. Eur J Orthod 2007;29:386-389.

[6] al Edris A, al Jabr A, Cooley RL, Barghi N. SEM evaluation of etch patterns by three etchants on three porcelains. J Prosthet Dent 1990;64:734-739.

[7] Roulet JF, Soderholm KJ, Longmate J. Effects of treatment and storage conditions on ceramic/composite bond strength. J Dent Res 1995;74:381-387.

[8] Bertolini JC. Hydrofluoric acid: a review of toxicity. J Emerg Med 1992;10:163-168.

[9] Fabianelli A, Pollington S, Papacchini F, Goracci C, Cantoro A, Ferrari M, van Noort R. The effect of different surface treatments on bond strength between leucite reinforced feldspathic ceramic and composite resin. J Dent 2010;38:39-43.

[10] Monticelli F, Toledano M, Osorio R, Ferrari M. Effect of temperature on the silane coupling agents when bonding core resin to quartz fiber posts. Dent Mater 2006;22:1024-1028.

[11] Turk T, Sarac D, Sarac YS, Elekdag-Turk S. Effects of surface conditioning on bond strength of metal brackets to all-ceramic surfaces. Eur J Orthod 2006;28:450-456.

[12] Abu Alhaija ES, Abu AIReesh IA, AIWahadni AM. Factors affecting the shear bond strength of metal and ceramic brackets bonded to different ceramic surfaces. Eur J Orthod 2010;32:274-280.

[13] Lung CY, Matinlinna JP. Aspects of silane coupling agents and surface conditioning in dentistry: an overview. Dent Mater 2012;28:467-477. 
[14] Nagayassu MP, Shintome LK, Uemura ES, Araujo JE. Effect of surface treatment on the shear bond strength of a resin-based cement to porcelain. Braz Dent J 2006;17:290-295.

[15] Özcan M, Valandro LF, Amaral R, Leite F, Bottino MA. Bond strength durability of a resin composite on a reinforced ceramic using various repair systems. Dent Mater 2009;25:1477-1483.

[16] Toroglu MS, Yaylali S. Effects of sandblasting and silica coating on the bond strength of rebonded mechanically retentive ceramic brackets. Am J Orthod Dentofacial Orthop 2008;134:181e181-187.

[17] Blum IR, Nikolinakos N, Lynch CD, Wilson NH, Millar BJ, Jagger DC. An in vitro comparison of four intra-oral ceramic repair systems. J Dent 2012;40:906-912.

[18] Gbureck U, Masten A, Probst J, Thull R. Tribochemical structuring and coating of implant metal surfaces with titanium oxide and hydroxyapatite layers. Mater Sci Eng C 2003;23:461-465.

[19] Pallesen U, van Dijken JW. An 8-year evaluation of sintered ceramic and glass ceramic inlays processed by the Cerec CAD/CAM system. Eur J Oral Sci 2000;108:239-246.

[20] 3M ESPE, 2011. Lava Ultimate CAD/CAM Restorative Technical Product Profile. St. Paul, 3M Espe Dental Products.

[21] Coldea A, Swain MV, Thiel N. Mechanical properties of polymer-infiltrated-ceramic-network materials. Dent Mater 2013;29:419-426.

[22] He LH, Swain M. A novel polymer infiltrated ceramic dental material. Dent Mater. 2011;27:527-534.

[23] Faul F, Erdfelder E, Buchner A, Lang AG. Statistical power analyses using G*Power 3.1: tests for correlation and regression analyses. Behav Res Methods 2009;41:1149-1160.

[24] Reynolds IR, von Fraunhofer JA. Direct bonding of orthodontic attachments to teeth: the relation of adhesive bond strength to gauze mesh size. Br J Orthod 1976;3:91-95.

[25] Thurmond JW, Barkmeier WW, Wilwerding TM. Effect of porcelain surface treatments on bond strengths of composite resin bonded to porcelain. J Prosthet Dent 1994;72:355-359.

[26] Bayram M, Yesilyurt C, Kusgoz A, Ulker M, Nur M. Shear bond strength of orthodontic brackets to aged resin composite surfaces: effect of surface conditioning. Eur J Orthod 2011;33:174-179.

[27] Schmage P, Nergiz I, Herrmann W, Ozcan M. Influence of various surface-conditioning methods on the bond strength of metal brackets to ceramic surfaces. Am J Orthod Dentofacial Orthop 2003;123:540546. 


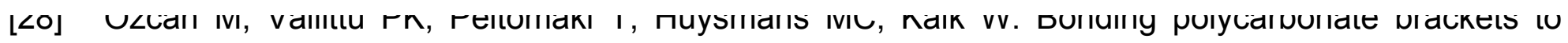
ceramic: effects of substrate treatment on bond strength. Am J Orthod Dentofacial Orthop 2004;126:220227.

[29] Ajlouni R, Bishara SE, Oonsombat C, Soliman M, Laffoon J. The effect of porcelain surface conditioning on bonding orthodontic brackets. Angle Orthod 2005;75:858-864.

[30] Alex G. Preparing porcelain surfaces for optimal bonding. Compend Contin Educ Dent 2008;29:324335.

[31] Monobond Plus, 2009. Scientific Documentation. Ivoclar Vivadent, Schaan, Liechtenstein.

[32] Belli R, Geinzer E, Muschweck A, Petschelt A, Lohbauer U. Mechanical fatigue degradation of ceramics versus resin composites for dental restorations. Dent Mater 2014;30:424-432.

[33] Della Bona A, Corazza PH, Zhang Y. Characterization of a polymer-infiltrated ceramic-network material. Dent Mater 2014;30:564-569.

[34] He LH, Swain MV. Nanoindentation derived stress-strain properties of dental materials. Dent Mater 2007;23:814-821.

[35] He LH, Swain MV. Enamel - a "metallic-like" deformable biocomposite. J Dent 2007;35:431-437.

[36] Smith GA, Mclnnes-Ledoux P, Ledoux WR, Weinberg R. Orthodontic bonding to porcelain-bond strength and refinishing. Am J Orthod Dentofacial Orthop 1988;94:245-252.

[37] Littlewood SJ, Mitchell L, Greenwood DC. A randomized controlled trial to investigate brackets bonded with a hydrophilic primer. J Orthod 2001;28:301-305.

[38] Fox NA, McCabe JF, Buckley JG. A critique of bond strength testing in orthodontics. Br J Orthod 1994;21:33-43. 


\section{Captions to tables and figures:}

\section{Tables:}

Table 1. Brands, types, manufacturers and batch numbers of the CAD/CAM materials used in this study.

Table 2. Results of two-way analysis of variance and Tukey`s tests $(\alpha=0.05)$.

Table 3. Mean shear bond strengths, standard deviations (SDs), minimum (Min) and maximum (Max) values, and confidence intervals (95\%) and Weibull parameters (95\% Cl) using maximum likelihood estimation, scale and shape values for each group $(n=17)$. *Same superscript letters in each column show homogeneous subsets $(\alpha=0.05)$. C: Control; CJ: CoJet+silanization; HF: Hydrofluoric acid+silanization. See Table 1 for material abbreviations.

Table 4. Distribution of Adhesive Remnant Index (ARI) scores after shear bond strength testing per experimental group (n, \%). Score 1: $90 \%$ of adhesive resin remained on the substrate surface; Score 2: $>10 \%$ but $<90 \%$ of adhesive resin remained on the substrate surface; Score $3:<10 \%$ of the adhesive remained on the substrate surface, Score 4: No adhesive resin remained on the substrate surface; Score 5: Cohesive failure in the substrate material.

\section{Figures:}

Figs.1a-f Representative scanning electron microphotographs showing different failure types a) Score 0 , b) Score 1, c) Score 2, d) Score 3, e) Score 4 and f) Score 5 from CAD/CAM material and surface conditioning combinations after bracket debonding ( ${ }^{*}$ indicates the resin cement; $\Rightarrow$ CAD/CAM material). See Table 4 for the description of the scores. 


\section{Tables:}

\begin{tabular}{|l|l|l|l|}
\hline Brands (Codes) & Type & Manufacturer & $\begin{array}{l}\text { Batch } \\
\text { No }\end{array}$ \\
\hline $\begin{array}{l}\text { Vita Mark II } \\
\text { (VM) }\end{array}$ & Feldspathic ceramic & $\begin{array}{l}\text { VITA Zahnfabrik, Bad } \\
\text { Säckingen, Germany }\end{array}$ & 34920 \\
\hline $\begin{array}{l}\text { IPS e.max CAD } \\
\text { (IP) }\end{array}$ & $\begin{array}{l}\text { Lithium disilicate glass } \\
\text { ceramic }\end{array}$ & $\begin{array}{l}\text { Ivoclar Vivadent, Schaan, } \\
\text { Liechtenstein }\end{array}$ & T36909 \\
\hline $\begin{array}{l}\text { Lava Ultimate } \\
\text { (LU) }\end{array}$ & Nanocomposite & $\begin{array}{l}\text { 3M ESPE, St. Paul, MN, } \\
\text { USA }\end{array}$ & N652052 \\
\hline $\begin{array}{l}\text { Vita ENAMIC } \\
\text { (VE) }\end{array}$ & $\begin{array}{l}\text { Polymer infiltrated } \\
\text { ceramic network }\end{array}$ & VITA Zahnfabrik & 41470 \\
\hline
\end{tabular}

Table 1. Brands, types, manufacturers and batch numbers of the CAD/CAM materials used in this study.

\begin{tabular}{|l|l|l|l|l|l|}
\hline Source of variation & $\begin{array}{l}\text { Type III Sum of } \\
\text { Squares }\end{array}$ & df & Mean Square & $\boldsymbol{F}$ ratio & $\boldsymbol{p}$ \\
\hline CAD/CAM Material & 1829.234 & 3 & 609.745 & 2.619 & 0.052 \\
\hline Surface Conditioning & 141765.273 & 2 & 70882.636 & 304.483 & 0.000 \\
\hline $\begin{array}{l}\text { CAD/CAM Material * Surface } \\
\text { Conditioning }\end{array}$ & 8142.555 & 6 & 1357.092 & 5.830 & 0.000 \\
\hline Error & 44697.007 & 192 & 232.797 & & \\
\hline Corrected Total & 196434.069 & 203 & & & \\
\hline
\end{tabular}

Table 2. Results of two-way analysis of variance and Tukey`s tests $(\alpha=0.05)$. 


\begin{tabular}{|c|c|c|c|c|c|c|c|c|c|}
\hline \multirow[b]{2}{*}{$\begin{array}{l}\text { Experimental } \\
\text { Groups }\end{array}$} & & \multirow[b]{2}{*}{ Mean } & \multirow[b]{2}{*}{ SD } & \multirow[b]{2}{*}{ Min } & \multirow[b]{2}{*}{ Max } & \multirow[b]{2}{*}{ Shape } & \multirow[b]{2}{*}{ Scale } & \multicolumn{2}{|c|}{ Confidence Interval } \\
\hline & & & & & & & & $\begin{array}{l}\text { Lower } \\
\text { Bound }\end{array}$ & Upper Bound \\
\hline \multirow[t]{3}{*}{ VM } & C & $5.3^{C}$ & 1.03 & 3.24 & 6.93 & 5.963 & 63.57 & 4.77 & 5.83 \\
\hline & CJ & $10.82^{A B}$ & 1.19 & 8.81 & 12.57 & 11.26 & 125.9 & 10.2 & 11.42 \\
\hline & HF & $9.81^{B}$ & 1.14 & 8.36 & 12.03 & 8.867 & 114.8 & 9.22 & 10.39 \\
\hline \multirow[t]{3}{*}{$\overline{\mathbf{I P}}$} & C & $4.73^{C}$ & 0.93 & 2.84 & 6.49 & 1.038 & 187.6 & 4.25 & 5.2 \\
\hline & CJ & $11.83^{\mathrm{A}}$ & 1.95 & 7.48 & 14.21 & 1.254 & 327.7 & 10.82 & 12.83 \\
\hline & HF & $10.7^{A B}$ & 1.98 & 7.31 & \begin{tabular}{|l|}
14.37 \\
\end{tabular} & 1.491 & 273.1 & 9.68 & 11.71 \\
\hline \multirow[t]{3}{*}{$\overline{\mathrm{LU}}$} & C & $6.02^{C}$ & 0.69 & 4.87 & 7.36 & 0.82 & 343.7 & 5.66 & 6.37 \\
\hline & CJ & $9.78^{B}$ & 1.61 & 7.63 & 12.39 & 1.589 & 234.7 & 8.94 & 10.6 \\
\hline & HF & $9.44^{\mathrm{B}}$ & 1.63 & 7.13 & 12.26 & 1.118 & 337.1 & 8.59 & 10.27 \\
\hline \multirow[t]{3}{*}{$\overline{\text { VE }}$} & C & $5.7^{\mathrm{C}}$ & 0.91 & 3.84 & 7.44 & 1.12 & 207.4 & 5.23 & 6.16 \\
\hline & CJ & $10.3^{A B}$ & 1.38 & 8.24 & 12.5 & 1.225 & 301.8 & 9.59 & 11.01 \\
\hline & HF & $10.93^{A B}$ & 1.33 & 8.58 & 13.45 & 1.827 & 244.4 & 10.25 & 11.61 \\
\hline
\end{tabular}

able 3. Mean shear bond strengths, standard deviations (SDs), minimum (Min) and maximum (Max) values, and confidence intervals (95\%) and Weibull parameters (95\% Cl) sing maximum likelihood estimation, scale and shape values for each group $(n=17)$. *Same superscript letters in each column show homogeneous subsets (alpha $=0.05)$. C: ontrol; CJ: CoJet+silanization; HF: Hydrofluoric acid+silanization. See Table 1 for material abbreviations. 


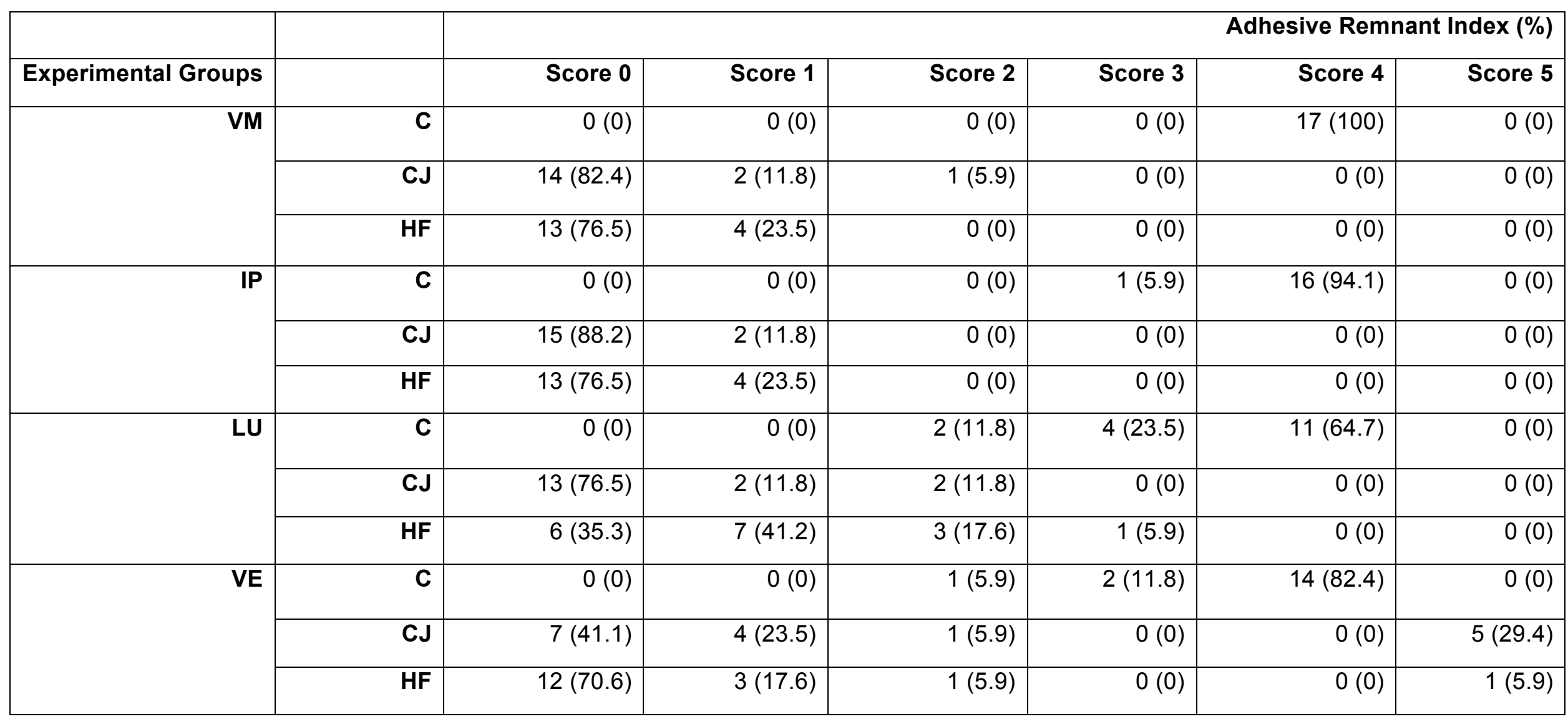

able 4. Distribution of Adhesive Remnant Index (ARI) scores after shear bond strength testing per experimental group (n, \%). Score 0: All adhesive remaining on he substrate surface; Score 1: $90 \%$ of adhesive resin remained on the substrate surface; Score $2:>10 \%$ but $<90 \%$ of adhesive resin remained on the substrate iurface; Score 3: $<10 \%$ of the adhesive remained on the substrate surface, Score 4: No adhesive resin remained on the substrate surface; Score 5: Cohesive ailure in the substrate material. 
Figures:

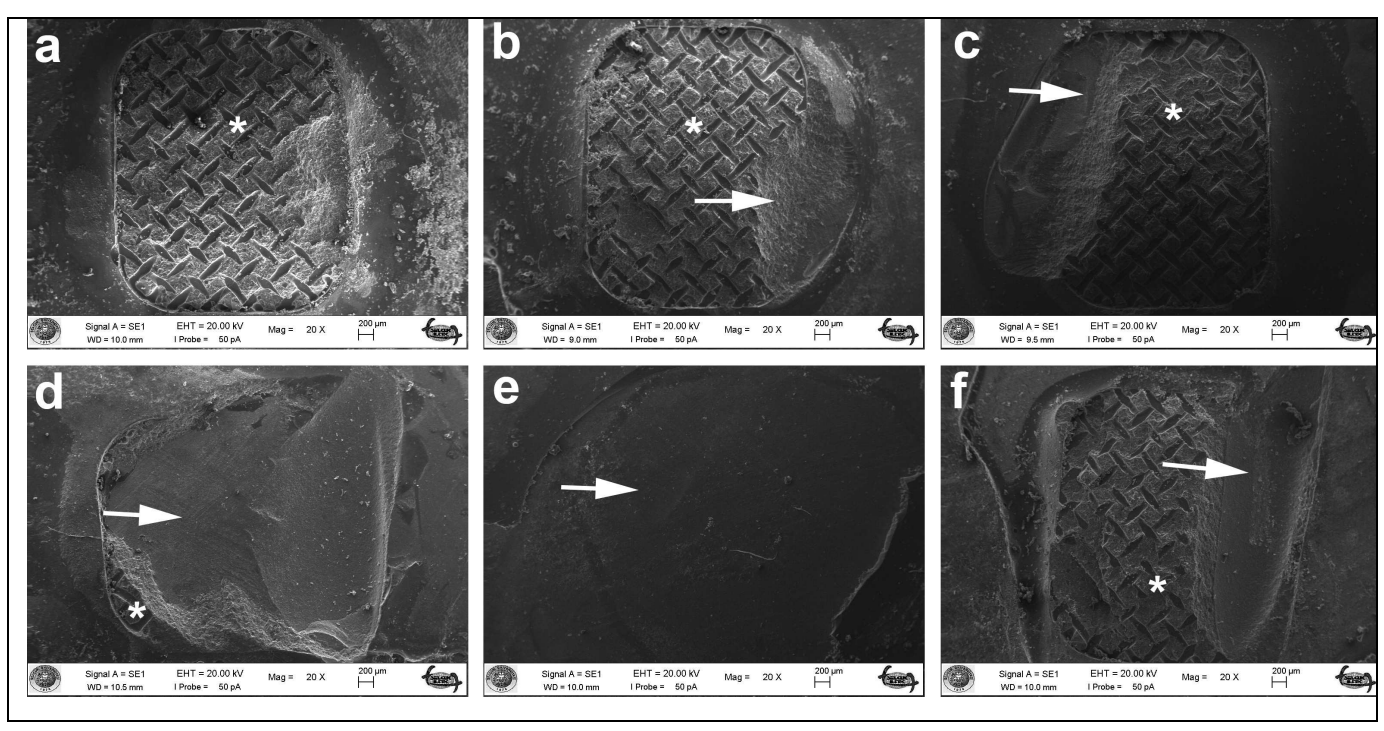

Figs.1a-f Representative scanning electron microphotographs showing different failure types a) Score 0, b) Score 1, c) Score 2, d) Score 3, e) Score 4,

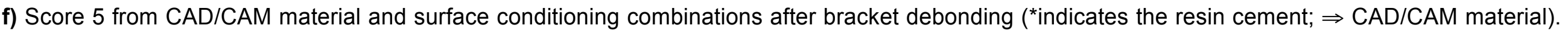
See Table 4 for the description of the scores 